\title{
Energy efficient routing protocols for wireless sensor networks: comparison and future directions
}

\author{
Murukesan Loganathan ${ }^{1 *}$, Thennarasan Sabapathy ${ }^{1}$, Nur Hafizah Ghazali ${ }^{1}$, R Badlishah Ahmad ${ }^{2}$ and Mohamed Nasrun \\ Osman $^{1}$ \\ ${ }^{1}$ Bioelectromagnetic (BioEM) Research Group, School of Computer and Communication Engineering, University Malaysia Perlis, \\ Pauh Putra, Perlis, Malaysia. ${ }^{2}$ Faculty of Informatics and Computing, Universiti Sultan Zainal Abidin (UniSZA), 22200 Besut, \\ Terengganu.
}

\begin{abstract}
Wireless sensor network consists of nodes with limited resources. Hence, it is important to design protocols or algorithms which increases energy efficiency in order to improve the network lifetime. In this paper, techniques used in the network layer (routing) of the internet protocol stack to achieve energy efficiency are reviewed. Usually, the routing protocols are classified into four main schemes: (1) Network Structure, (2) Communication Model, (3) Topology Based, and (4) Reliable Routing. In this work, only network structure based routing protocols are reviewed due to the page constraint. Besides, this type of protocols are much popular among the researchers since they are fairly simple to implement and produce good results as presented in this paper. Also, the pros and cons of each protocols are presented. Finally, the paper concludes with possible further research directions.
\end{abstract}

\section{Introduction}

Wireless sensor networks (WSN) are result of recent advances in micro-electro-mechanical system (MEMS) technology which enables the manufacturing of small and low cost sensors technically and economically feasible. Sensor nodes were initially designed and developed for the use of military and defense applications. In fact, they were used during Vietnam War to detect enemy soldiers in dense forest [1]. However, those sensors had serious limitations such as high energy consumption, bulky size and very basic network capability. Fast forward few decades and now sensor nodes are much smaller in size but with high computational capability as compared to the earlier nodes. Figure 1 shows basic building blocks of a typical sensor node.

The microcontroller is designed to consume ultra-low power since the attached battery is usually nonreplaceable once the sensor nodes are deployed. In addition, the size of the battery also limited since sensor nodes are required be small [2]. It also has limited storage capacity. Moreover, IEEE 802.15.4 compatible radio is used since the protocol is designed for devices with limited energy.

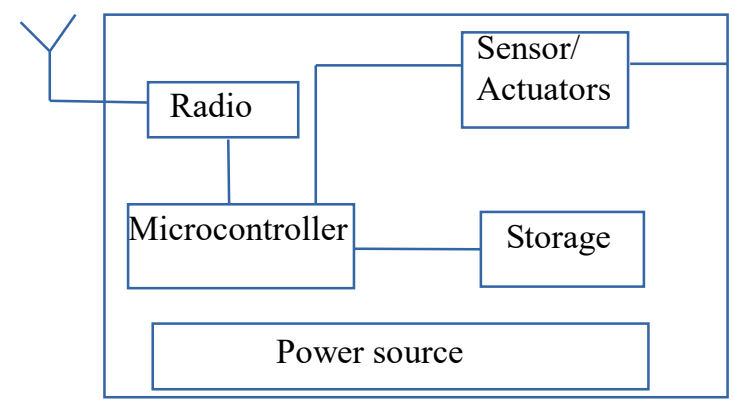

Fig. 1. Building blocks of a typical sensor node.

It is evident from the design itself that the sensor nodes are expected to be energy efficient in order to maximize the lifetime. Hence, it is only natural that there are plethora of energy efficient routing protocols in the literature to support data delivery to the destination with minimal energy consumption. However, these routing protocols are often application- and network architecture-specific since WSNs have been used in many diverse applications. Based on the environment that sensor nodes are being deployed, there are multiple types of WSNs such as wireless body area network (WBAN), wireless multimedia sensor network (WMSN) and etc.

Nevertheless, there are some problems which are common for any type of WSN. For instance, although 
current generation sensor nodes are designed to be energy efficient, lifetime of sensor nodes is still one of the main concerns [2]. Also, lifetime of sensor nodes is dependent on both hardware and software implementations. Even though the components that assemble the sensor node save energy, inefficient protocols may lead to early death of the nodes. Energy efficiency can be achieved in WSN through implementation of efficient algorithms and protocols at various network stack level [3].

As shown in the figure 2, a sensor node protocol stack consists of physical layer, data link layer, network layer, transport layer, application layer, power management plane, mobility management plane and task management plane [4]. Physical layer is responsible for frequency selection, carrier frequency generation, signal detection, modulation, and data encryption. The data link layer is responsible for multiplexing of data streams, date frame detection, and medium access and error control. Besides, the network layer is responsible for routing while transport layer provides transmission protocol and congestion control. Also, application layer includes the main application and several management functionalities. Finally, the power, mobility, and task management planes monitor the power, movement, and task distribution among the sensor nodes. These planes help the sensor nodes to coordinate the sensing task and lower the overall power consumption.

The main contribution of this paper is to provide a survey (not exhaustive) from 2011 to 2016 on the energy efficient routing protocols for WSNs. This is so that new researchers can readily know about the current trends in the field. Even though there are several surveys in the literature as shown in Table 1, this work approaches routing protocols from an energy efficiency perspective. Also, we discuss the pros and cons of each protocols by making comparison between them using related metrics (scalability, mobility and robustness).

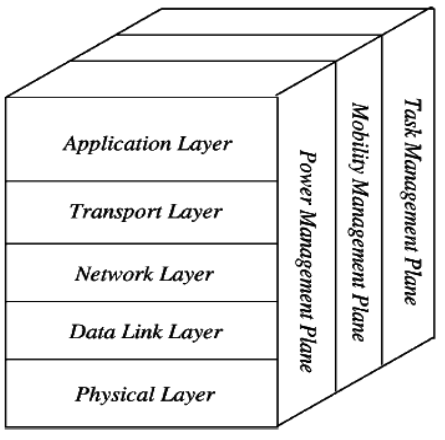

Fig. 2. Network protocol stack of sensor nodes [4]

Besides, we also suggest few future research directions for researchers based on the identified limitations in the present routing protocols.

The main contribution of this paper is to provide a survey (not exhaustive) from 2011 to 2016 on the energy efficient routing protocols for WSNs. This is so that new researchers can readily know about the current trends in the field. Even though there are several surveys in the literature as shown in Table 1, this work approaches routing protocols from an energy efficiency perspective. Also, we discuss the pros and cons of each protocols by

\section{Classification of routing protocols}

In this paper, we employed the routing protocol classification (Figure 3) as proposed by Pantazis et al making comparison between them using related metrics (scalability, mobility and robustness). Besides, we also suggest few future research directions for researchers based on the identified limitations in the present routing protocols.

This paper is organized as follow: In section 2, classification of routing protocols is explained. In section 3 , network structure based routing protocols are reviewed. In section 4, possible future research directions are presented. Finally, this paper is concluded in section 5 .

[1]. It should be noted that some of the protocols described in following section, may fall under one or more of the routing categories shown in the figure 3 , but only the protocols which has network structure scheme as its foundation is discussed here.

Table 1. Previous surveys.

\begin{tabular}{ccc}
\hline Authors & Characteristic & Time Period \\
\hline$[5]$ & WSN routing & $1988-2004$ \\
{$[6]$} & $\begin{array}{c}\text { Multipath } \\
\text { routing }\end{array}$ & $1993-2011$ \\
& QoS routing & $1990-2012$ \\
{$[7]$} & WSN routing & $2004-2011$ \\
{$[1]$} & WBAN survey & $2000-2013$ \\
{$[8]$} & Real-time QoS & $2000-2015$ \\
{$[9]$} & routing \\
\hline
\end{tabular}




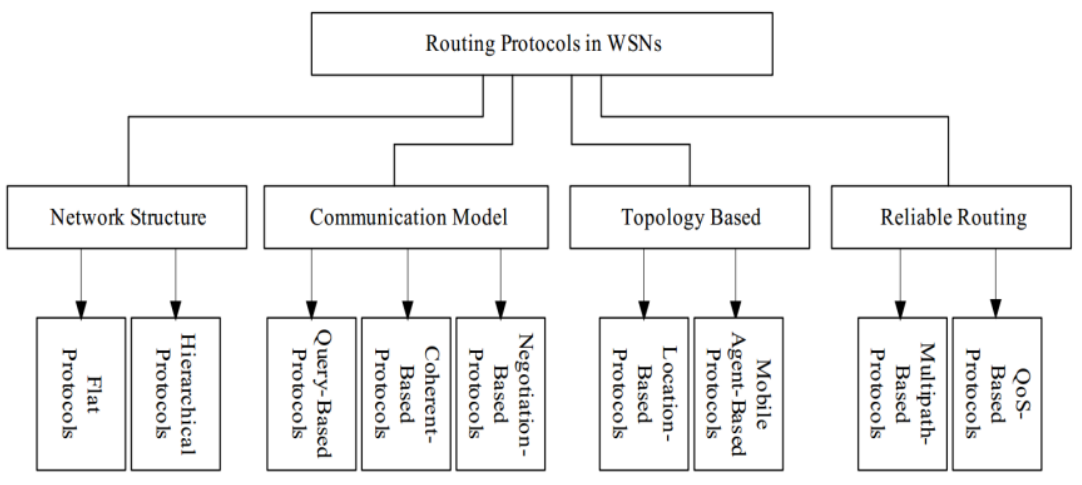

Fig. 3. Classification of WSN routing protocols [1].

Also, tables 2 and 3 summarizes the advantages and disadvantages of each protocol described in this paper. In addition, some metrics for each protocol are presented. These metrics are as follow:

- Scalability - The scalability of a protocol refers to the ability of the protocol to maintain its performance for both small and large networks.

- Mobility - This metric denotes the ability of the protocol to manage mobile nodes. Low mobility means the protocol either does not consider the mobility all together or very limited number of mobile nodes in very slow speed are allowed. Good mobility is when the protocol supports large number of mobile nodes with moderate speed.

- $\quad$ Route Metric - This metric denotes the form of the routing selected to send data from source to destination in such a way that the path taken to send the data is most efficient. Besides, the criteria to choose a path varies across each protocols as shown in these tables.

- Robust - This metric denotes the ability of the protocol to cope up with sudden changes in the network such as topological and link quality changes. The protocol is not robust if it does not consider both the topological and link quality changes. If either one is considered, then it is a moderately robust protocol. It is rated good when both the topological and link changes were considered in the protocol.

\section{Routing protocols based on network structure}

\subsection{Flat protocols}

The first category of routing protocols is the multi-hop flat routing protocols. In flat networks, each node typically plays the same role and sensor nodes collaborate together to perform the sensing task. In the rest of this subsection, these protocols are summarized and their limitations are highlighted.

Amiri et al. had studied the feasibility of using fuzzy logic and ant colony optimization (FACOR) for an energy efficient routing in flat WSN architecture [10]. Basically, the ants were used to find best candidate routes to base station while fuzzy value is used by the ants to determine best next hop node. Route that have least number of hops and highest fuzzy value is selected as optimal path. The FACOR protocol was compared with ad-hoc on-demand distance vector (AODV) protocol from the literature in terms of energy efficiency, complexity, end-to-end delay and reliability. Proposed protocol proven to be $40 \%$ more energy efficient as compared to AODV in same scenario. However, the setup time for FACOR is higher than as for the AODV protocol. Besides, the ant colony would take longer time to stabilize in large scale WSNs. In addition, this protocol is not suitable for WSNs with heterogeneous nodes and it is not fault-tolerant.

Ghadimi et al. had proposed opportunistic routing in wireless sensor networks (ORW) routing protocol targeting applications with low duty cycles [11]. The protocol maintains a so called expected duty cycle (EDC) metric for all the neighboring nodes. Node with highest EDC is selected to forward the packet to the sink. Unlike other protocols, ORW makes use of both the reliable and unreliable links for data forwarding. The proposed ORW was compared with collection tree protocol (CTP) protocol in terms of delay, reliability, transmission count and radio duty cycle. Using test bed implementation, ORW was found to reduce duty cycles on average by $50 \%$ and delays by $30 \%$ to $90 \%$ while achieving reliability and transmission counts similar to the CTP protocol. However, ORW has several limitations as follow. First, it is not applicable for network with high throughput requirement. Also, the protocol does not support mesh routing. In addition, mobility factor was overlooked.

A routing protocol based on routing by energy and link quality (REL) for internet of things applications is proposed in [12]. The protocol maintains a metric called link quality index (LQI) for each nodes as shown in figure 4. Route which has lesser number of weak links with minimum hop counts and more residual energy is selected as optimal path. The proposed routing protocol was compared with link quality-based lexical routing metric (LABILE) and AODV protocols in terms of energy efficiency, PDR, and latency. In large scale network simulation with high node density (200 nodes), REL increases network lifetime by up to $26.6 \%$, latency by $17.9 \%$ and PDR by $12 \%$, as compared to both AODV and LABILE. However, only uniform distribution of nodes is considered in REL. In real life, nodes are rarely 
distributed in uniform manner. Since inter-packetinterval of $2 \mathrm{~s}$ is used, this protocol is ill-suited for real- time multimedia content applications which are delay sensitive such as streaming and monitoring.

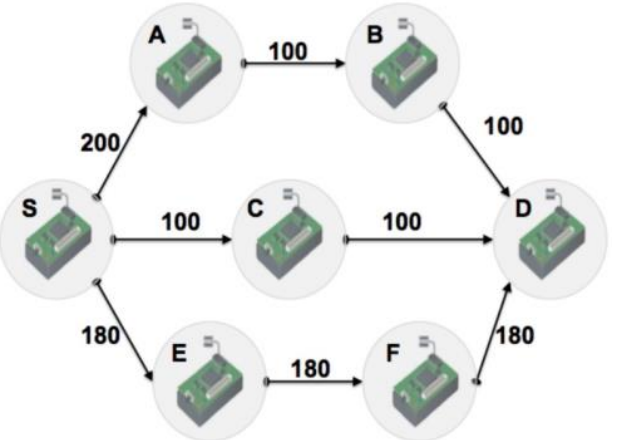

Fig. 4. Nodes with calculated LQI in REL protocol [12].

Jain and Reddy had proposed an orthogonal directional proactive-reactive routing protocol (ODPRRP) using ant colony optimization and fuzzy logic [13]. The protocol uses directed fuzzy vertex and edge graph of WSN and ant colony to find optimal path to the sink node. Also, nodes are classified into two which are the normal and proactive nodes. The proactive nodes are used for load balancing purposes along with its normal operation. The OD-PRRP was compared with energy aware routing (EARQ), enhanced AODV (EAODV) and energy efficient ant-based routing (EEABR) protocols from literature. Besides, it was evaluated using mobile nodes to evaluate its performance in dynamic environment. OD-PRRP is $57.9 \%, 114.3 \%$ and $160.9 \%$ better in energy efficiency as compared to EAODV, EEABR and EARQ, respectively. But, the protocol was not evaluated in heterogeneous networks. Also, the reported performance figure cannot be verified using current generation sensor nodes since they all use omnidirectional antenna in contrast to directional antenna as used in this simulation.

In table 2, comparison between flat routing schemes using various metrics such as scalability, mobility, realtime support and robustness of the algorithms is given.

Table 2. Comparison of Flat Routing Schemes

\begin{tabular}{|c|c|c|c|c|c|c|c|}
\hline Scheme & Pros & Cons & Scalability & Mobility & Route Metric & Robust & Real-Time \\
\hline FACOR & $\begin{array}{l}\text { Energy efficient, reliable } \\
\text { and have good end-to-end } \\
\text { delay. }\end{array}$ & $\begin{array}{l}\text { No mobility and fault- } \\
\text { tolerance }\end{array}$ & Good & $\begin{array}{c}\text { Not } \\
\text { Considered }\end{array}$ & $\begin{array}{l}\text { Minimum hop with } \\
\text { highest fuzzy value }\end{array}$ & Low & Good \\
\hline ORW & $\begin{array}{l}\text { Decreases delay and duty- } \\
\text { cycle without sacrificing } \\
\text { energy efficiency and } \\
\text { reliability. }\end{array}$ & $\begin{array}{l}\text { Not applicable for } \\
\text { network with high } \\
\text { throughput } \\
\text { requirement. }\end{array}$ & Good & Good & $\begin{array}{l}\text { Expected duty-cycle } \\
\text { (EDC) }\end{array}$ & Good & Good \\
\hline REL & $\begin{array}{l}\text { Energy efficiency and load } \\
\text { balancing using end-to-end } \\
\text { link quality for IOT } \\
\text { applications. }\end{array}$ & $\begin{array}{l}\text { Mobility factor was } \\
\text { overlooked. }\end{array}$ & Good & $\begin{array}{c}\text { Not } \\
\text { Considered }\end{array}$ & $\begin{array}{l}\text { Threshold (link } \\
\text { quality, residual } \\
\text { energy and hop } \\
\text { counts) }\end{array}$ & Moderate & Good \\
\hline $\begin{array}{l}\text { OD- } \\
\text { PRRP }\end{array}$ & $\begin{array}{l}\text { OD-PRRP reduces control } \\
\text { overhead without } \\
\text { compromising cost and } \\
\text { energy efficiency }\end{array}$ & $\begin{array}{l}\text { Directional antenna is } \\
\text { not currently available } \\
\text { on motes. }\end{array}$ & Good & Good & Orthogonal direction & Good & Good \\
\hline
\end{tabular}

\subsection{Hierarchical protocols}

In hierarchical routing, nodes are grouped into clusters and every cluster has a cluster head. In the rest of this subsection, hierarchical protocols are summarized and their limitations are highlighted.

Shah et al. proposed scaling hierarchical clustering and energy aware routing (SHEAR) protocol with the aim to improve network lifetime by using power efficient and scalable clustering together with an energy aware path selection scheme [14]. This protocol is based on the scaling hierarchical power efficient routing (SHPER) by [15]. In this protocol, WidestShortestPath algorithm is used to find all the paths in the network which have highest residual energy after the $\mathrm{CH}$ election process. From these set of paths, a path which minimizes energy consumption is selected for routing. The proposed SHEAR protocol was compared with SHPER from the literature in terms of number of transmission, energy consumption and the number of nodes alive over time. SHEAR was found to increase the network lifetime by $23 \%$ as compared to the SHPER protocol. However, the protocol is not resilient to topological changes. Also, the cluster formation and $\mathrm{CH}$ election for every transmission round is not energy efficient.

In [16], authors had proposed the low loss energy aware protocol (LLEAP) to address the lack of load balancing and topology updating mechanism in the original energy aware protocol (EAP). In this protocol, after the $\mathrm{CH}$ election and cluster formation state, $\mathrm{CHs}$ form a collection tree rooted at the sink node. The protocol also incorporates some modifications into the main protocol so that the data packets are not routed to a dead node. The proposed LLEAP was compared with EAP protocol from the literature in terms of throughput, 
delay and energy efficiency. LLEAP was found to improve energy efficiency by $7.8 \%$ as compared to the EAP protocol. The improvement in network lifetime is not significant. Besides, it was achieved by trading-off the latency metric.

Kong et al. proposed a novel instantaneous clustering protocol (ICP) that groups sensor nodes into single-hop clusters in a parallel manner [17]. In this protocol, $\mathrm{CHs}$ are locally determined by the pre-assigned probability and its present status. Besides, to save energy, ICP get rids of acknowledgment mechanism and only $\mathrm{CHs}$ are allowed to contend for transmission period. This period is elaborately derived to guarantee the connectivity. The proposed protocol was evaluated using both test bed and simulation. It was compared with LEACH, energy constrained minimum dominating set based efficient clustering (ECDS), balanced clustering algorithm with distributed self-organization for wireless sensor networks (DSBCA) and fault-tolerant energy efficient clustering (FT-EEC) protocols from the literature in terms of setup time and energy efficiency. Performance results demonstrate that ICP significantly outperforms existing clustering methods by reducing up to 55\%-time consumption and $89 \%$ message overheads for energysaving.

In [18], authors had proposed an evolutionary algorithm based clustering and routing protocol (ERP) for heterogeneous wireless sensor networks in order to optimize the stability period and network lifetime. This was achieved by formulating a new fitness function that incorporates two clustering aspects, namely, cohesion and separation error. Readers are encouraged to refer page 4 of original manuscript for a detailed description on used evolutionary algorithm parameters (mutation, crossover, probabilities of mutation etc.). Routing of data packets is done in multi-hop fashion after the clusters were formed. Proposed ERP protocol was compared with stable election protocol (SEP), LEACH and hybrid cluster routing (HCR) protocols from the literature in terms of stability period and network lifetime. The ERP protocol produced $42 \%, 62 \%$ and $3 \%$ lifetime improvement as compared to LEACH, SEP, and HCR protocols, respectively. However, the stability period of ERP is slightly lower than SEP but better than LEACH and HCR protocols. Besides, the protocol can be further optimized by introducing optimal route selection policy and load balancing. Nevertheless, the complexity of the EA algorithms makes it a tough candidate for current generation sensor nodes which are computationally challenged [19].

Building on ERP, Attea and Khalil proposed stable aware evolutionary routing protocol (SAERP) protocol which increases the stability period (time for first node to die) of WSN while reducing the instability period (time from the death of first node until the last node) using an evolutionary algorithm (EA) based approach [20]. The proposed SAERP was compared with LEACH and SEP protocols from the literature in terms of stability, instability periods and throughput. SAERP prolongs the stability period for more than hundreds of rounds as compared to both the SEP and LEACH protocols. Besides, it has very steep instability period which shows its excellent load balancing capability. In addition, SAERP provides more throughput per energy dissipation as compared to the SEP and LEACH protocols. However, the time complexity of this protocol renders it impractical for sensor nodes.

Roy had proposed energy aware cluster based routing scheme (EACBRS) which selects energy efficient route to the sink while providing congestion control mechanism to minimize the re-transmissions [21]. In this protocol, after the $\mathrm{CH}$ election process, nodes send the sensed data to their cluster heads by following the congestion control mechanism where data are buffered in the memory for a period. Thus, no packets are dropped or number of re-transmissions are reduced considerably. In addition, $\mathrm{CHs}$ aggregate the data and send it to the sink to save energy. The proposed EACBRS was compared with distributed energy efficient clustering (DEEC) and energy efficient hybrid clustering (EEHC) protocols from the literature in terms of network lifetime and throughput. Average residual energy of nodes in EACBRS protocol is significantly higher than as compared to DEEC and EEHC protocols. However, the stability period of proposed protocol is very low as compared to other state of the art protocols such as SAERP and ERP in the literature. Besides, the protocol does not consider nodes mobility.

In table 3, comparison of hierarchical routing schemes using various metrics such as scalability, mobility, real-time support and robustness of the algorithms is given.

Table 3. Comparison of Hierarchical Routing Scheme

\begin{tabular}{|c|c|c|c|c|c|c|c|}
\hline Scheme & Pros & Cons & Scalability & Mobility & Route Metric & Robust & Real-Time \\
\hline SHEAR & $\begin{array}{l}\text { Two level cluster formation } \\
\text { with energy efficient path } \\
\text { selection. }\end{array}$ & $\begin{array}{l}\text { Waste of energy due to } \\
\text { clustering and } \mathrm{CH} \\
\text { selection for every } \\
\text { round. }\end{array}$ & Good & $\begin{array}{c}\text { Not } \\
\text { Considered }\end{array}$ & $\begin{array}{l}\text { Residual energy } \\
\text { with least cost } \\
\text { path }\end{array}$ & Low & $\begin{array}{c}\text { Not } \\
\text { Considered }\end{array}$ \\
\hline LLEAP & $\begin{array}{l}\text { Three phase cluster and tree } \\
\text { formation for balanced } \\
\text { routing. }\end{array}$ & $\begin{array}{l}\text { Insignificant } \\
\text { improvement in } \\
\text { network lifetime. }\end{array}$ & Good & $\begin{array}{c}\text { Not } \\
\text { considered }\end{array}$ & Multi-hop & Moderate & Moderate \\
\hline ICP & $\begin{array}{l}\text { Efficient clustering with } \\
\text { fast setup time and lower } \\
\text { overheads. }\end{array}$ & $\begin{array}{l}\text { Delay and reliability } \\
\text { are not investigated. }\end{array}$ & Good & $\begin{array}{c}\text { Not } \\
\text { considered }\end{array}$ & Multi-hop & Moderate & $\begin{array}{c}\text { Not } \\
\text { Considered }\end{array}$ \\
\hline ERP & $\begin{array}{l}\text { Efficient cluster formation } \\
\text { using cohesion and cluster } \\
\text { separation metrics. }\end{array}$ & $\begin{array}{l}\text { Stability period is not } \\
\text { optimal. }\end{array}$ & Good & $\begin{array}{c}\text { Not } \\
\text { Considered }\end{array}$ & Multi-hop & Low & $\begin{array}{c}\text { Not } \\
\text { Considered }\end{array}$ \\
\hline SAERP & Clustering which increases & Highly complex & Good & Not & Multi-hop & Low & Not \\
\hline
\end{tabular}




\begin{tabular}{|c|c|c|c|c|c|c|c|}
\hline & $\begin{array}{l}\text { stability period while } \\
\text { decreasing the instability } \\
\text { period. }\end{array}$ & $\begin{array}{l}\text { algorithm which is not } \\
\text { feasible for sensor } \\
\text { nodes. }\end{array}$ & & Considered & & & Considered \\
\hline EACBRS & $\begin{array}{l}\text { Energy efficient route } \\
\text { selection with congestion } \\
\text { control. }\end{array}$ & $\begin{array}{l}\text { Very short stability } \\
\text { period. }\end{array}$ & Good & $\begin{array}{c}\text { Not } \\
\text { Considered }\end{array}$ & $\begin{array}{l}\text { Weight (residual } \\
\text { energy and } \\
\text { distance) }\end{array}$ & Low & $\begin{array}{c}\text { Not } \\
\text { Considered }\end{array}$ \\
\hline
\end{tabular}

\section{Future research directions}

In the recent years a large number of energy efficient routing protocols for the WSNs have been developed. However, there is still a lot of work that has to be done, not only in the area of energy efficiency but also, in other areas. In this subsection, some ideas and interesting avenues for routing protocols are elaborated.

\section{- $\quad$ Mobility support}

Majority of the reviewed routing protocols in this paper assume that nodes in the network are static. However, recently, there is an increased interest in applications that support the mobility of the users. The topological changes due to mobility and expected minimum reliability must be considered when designing mobility aware routing protocols.

- Real life performance evaluation using test beds or deployment

Most of the reviewed protocols were evaluated through simulations only. However, it is important to evaluate these protocols in real environments.

\section{- Load balancing}

Most of the reviewed protocol in this paper lacks load balancing capability. This leads to premature dead of a portion of the network.

\section{References}

[1] N. A. Pantazis, S. A. Nikolidakis, and D. D. Vergados, "Energy-Efficient Routing Protocols in Wireless Sensor Networks: A Survey," IEEE Commun. Surv. Tutorials, vol. 15, no. 2, pp. 551591, 2013.

[2] I. F. Akyildiz, W. Su, Y. Sankarasubramaniam, and E. Cayirci, "Wireless sensor networks: a survey," Comput. Networks, vol. 38, no. 4, pp. 393-422, 2002.

[3] R. Mahapatra, Y. Nijsure, G. Kaddoum, N. U. Hassan and C. Yuen, "Energy Efficiency Tradeoff Mechanism Towards Wireless Green Communication: A Survey", IEEE Communications Surveys and Tutorials, Jan 2016, pp. $686-705$.

[4] I. F. Akyildiz and M. C. Vuran, Wireless Sensor Networks, vol. 1. Wiley, 2010.
- Cross-layer cognitive radio based routing

Even though plenty of researches had been done on medium access control (MAC) for cognitive radio (CR) [22], [23], cognitive aware routing is still a fresh topic.

\section{Conclusion}

Wireless sensor networks had matured to a point where it has found its usage in civilian applications from pure military based applications in the past. However, energy efficiency is still one of the major concerns with the current generation sensor nodes as it had been in the past. Hence, various efforts had been taken to tackle this issue either through hardware or software optimizations. This paper focuses on reviewing efforts on the software side, specifically, in the network layer of the sensor nodes.

In this paper, we focus on the network structure based energy efficient routing protocols that have been developed for WSNs. Also, we presented pros and cons each protocols. In addition, possible future research directions were pinpointed as a guidance for researchers.

\section{Conflict of Interest}

The authors declare that there is no conflict of interests regarding the publication of this paper.

\section{Acknowledgment}

This work is not funded by any government or private organizations.

[5] Al-Karaki, J. N. and Kamal, A. E. (2004) 'Routing Techniques in Wireless Sensor Networks: A Survey', IEEE Wireless Communications, 11(6), pp. 6-28. doi: 10.5120/16346-5685.

[6] Jayashree, A., Biradar, G. S. and Mytri, V. D. (2012) 'Review of Multipath Routing Protocols in Wireless Multimedia Sensor Network -A Survey', International Journal of Scientific \& Engineering Research, 3(9), pp. 1-9.

[7] Ehsan, S. and Hamdaoui, B. (2012) 'A survey on energy-efficient routing techniques with QoS assurances for wireless multimedia sensor networks', IEEE Communications Surveys and Tutorials, 14(2), pp. 265-278.

[8] Movassaghi, S., Abolhasan, M., Lipman, J., Smith, D. and Jamalipour, A. (2014) 'Wireless 
Body Area Networks: A Survey', Ieee Communications Surveys and Tutorials, 16(3), pp. 1658-1686.

[9] Alanazi, A. and Elleithy, K. (2015) 'Real-Time QoS Routing Protocols in Wireless Multimedia Sensor Networks: Study and Analysis', Sensors, 15(9), pp. 22209-22233.

[10] E. Amiri, H. Keshavarz, M. Alizadeh, M. Zamani, and T. Khodadadi, "Energy efficient routing in wireless sensor networks based on fuzzy ant colony optimization," Int. J. Distrib. Sens. Networks, p. 17, 2014.

[11] E. Ghadimi, O. Landsiedel, P. Soldati, S. Duquennoy, and M. Johansson, "Opportunistic Routing in Low Duty-Cycled Wireless Sensor Networks," ACM Trans. Embed. Comput. Syst., vol. 0 , no. 0 , pp. 1-35, 2014.

[12] K. Machado, D. Rosário, E. Cerqueira, A. Loureiro, A. Neto, and J. de Souza, "A Routing Protocol Based on Energy and Link Quality for Internet of Things Applications," Sensors, vol. 13, no. 2, pp. 1942-1964, 2013.

[13] A. Jain and B. V. R. Reddy, "Ant Colony Optimization Based Orthogonal Directional Proactive-Reactive Routing Protocol for Wireless Sensor Networks," Wirel. Pers. Commun., vol. 85, no. 1, pp. 179-205, 2015.

[14] M. A. Shah, G. Abbas, A. B. Dogar, and Z. Halim, "Scaling hierarchical clustering and energy aware routing for sensor networks," Complex Adapt. Syst. Model., vol. 3, no. 1, p. 5, 2015.

[15] D. Kandris, P. Tsioumas, A. Tzes, G. Nikolakopoulos, and D. D. Vergados, "Power conservation through energy efficient routing in wireless sensor networks," Sensors, vol. 9, no. 9, pp. 7320-7342, 2009.

[16] B. M. Mohammad El-Basioni, S. M. Abd ElKader, H. S. Eissa, and M. M. Zahra, "An optimized energy-aware routing protocol for wireless sensor network," Egypt. Informatics J., vol. 12, no. 2, pp. 61-72, 2011.

[17] L. Kong et al., "ICP: Instantaneous clustering protocol for wireless sensor networks," Comput. Networks, vol. 101, pp. 144-157, 2016.

[18] B. Attea and E. Khalil, "A new evolutionary based routing protocol for clustered heterogeneous wireless sensor networks," Appl. Soft Comput., vol. 12, pp. 1950-1957, 2012.

[19] K. Lehre, "Time-complexity Analysis of Evolutionary Algorithms.” pp. 1-60, 2011.

[20] E. A. Khalil and B. A. Attea, "Stable-Aware Evolutionary Routing Protocol for Wireless Sensor Networks," Wirel. Pers. Commun., vol. 69, pp. 1799-1817, 2013.

[21] S. Roy, "Energy Aware Cluster based Routing Scheme for Wireless Sensor Network," Found. Comput. Decis. Sci., vol. 40, no. 3, pp. 203-222, 2015.

[22] A. De Domenico, E. Calvanese Strinati, and M. G. Di Benedetto, "A survey on MAC strategies for cognitive radio networks," IEEE Commun. Surv. Tutorials, vol. 14, no. 1, pp. 21-44, 2012.

[23] C. Cormio and K. R. Chowdhury, "A survey on MAC protocols for cognitive radio networks," Ad Hoc Networks, vol. 7, no. 7, pp. 1315-1329, 2009. 